\title{
COVID-19 pandemic and systemic autoinflammatory diseases management: a cross-sectional survey
}

\author{
$\mathrm{Na} \mathrm{Wu}{ }^{1} \cdot$ Mengzhu Zhao ${ }^{1} \cdot \mathrm{Di} \mathrm{Wu}^{1} \cdot \mathrm{Keyi} \mathrm{Yu}^{2} \cdot$ Min Shen $^{1} \odot$ \\ Received: 18 February 2021 / Accepted: 19 March 2021 / Published online: 12 April 2021 \\ (c) The Author(s), under exclusive licence to Springer-Verlag GmbH Germany, part of Springer Nature 2021
}

Since December 2019, coronavirus disease 2019 (COVID19) caused by severe acute respiratory syndrome-coronavirus 2 (SARS-CoV-2) infection has spread all over the world. On 30th January 2020, World Health Organization (WHO) officially declared the COVID-19 epidemic as a public health emergency of international concern [1]. The pandemic has posed a challenge regarding the routine health care to patients with chronic diseases [2]. Systemic autoinflammatory diseases (SAIDs) are a group of rare disorders which have chronic trajectories and share characteristics of periodic inflammation, demanding long-term disease management [3]. We aim to investigate the impact of COVID-19 outbreak on real-word healthcare of patients with SAIDs in China.

From April 2020 to May 2020, we enrolled patients who were diagnosed with SAIDs and followed up in our tertiary medical center to finish the designed questionnaire. This study is approved by Ethics Committee of Peking Union Medical College Hospital. We sent out the link of the questionnaire to 122 SAIDs patients through E-mail and WeChat (a communication application similar to "WhatsApp"). If

$\mathrm{Na} \mathrm{Wu}$ and Mengzhu Zhao contributed equally to the work.

Keyi Yu

yukeyi009@126.com

$\square$ Min Shen

shenmpumch@163.com

1 Department of Rheumatology and Clinical Immunology, Peking Union Medical College Hospital, Chinese Academy of Medical Sciences and Peking Union Medical College, National Clinical Research Center for Dermatologic and Immunologic Diseases (NCRC-DID), Key Laboratory of Rheumatology and Clinical Immunology, Ministry of Education, No.1 Shuaifuyuan, Dongcheng District, Beijing 100730, China

2 Department of Orthopaedic Surgery, Peking Union Medical College Hospital, Chinese Academy of Medical Sciences and Peking Union Medical College, No.1 Shuaifuyuan, Dongcheng District, Beijing 100730, China without reply, we further contacted them through telephone for their answers to the questionnaire. Finally, a total of 86 SAIDs patients completed the questionnaire, and the response rate was $70.5 \%$ (86/122). These patients were from 22 provinces and municipalities, mainly in western, central, and northern China. Their demographic characteristics and clinical information are listed in Table 1.

First of all, COVID-19 was ruled out in our participants since they had no typical symptoms and with the negative results of SARS-CoV-2 via reverse transcription polymerase chain reaction testing. During COVID-19 pandemic, $17(19.5 \%)$ patients with SAIDs had been hospitalized due to their disease activities. Some patients reported clinical symptoms of SAIDs, such as arthritis (40.7\%), fever (39.5\%), myalgia (27.9\%), fatigue (18.6\%), etc. Overall, 70 (80.5\%) patients did not complete their follow-up since the COVID-19 outbreak. There were three (3.5\%) patients who visited our center, seven (8.0\%) patients received consultations via E-mails, and seven (8.0\%) patients visited local outpatient clinics. Among those patients with no follow-up visits, $26(37.1 \%)$ deemed the pandemic as a noteworthy factor to impact their hospital visits. Only 33 (37.9\%) patients held the opinion that the pandemic did not pose a challenge to their disease treatment and follow-ups. In the meantime, the rest of these patients thought the pandemic did have an impact on their disease treatment and follow-ups to different extents: slightly $(24 / 87,27.6 \%)$, moderately $(16 / 87,18.4 \%)$, severely $(10 / 87,11.5 \%)$, and extremely $(4 / 87,4.6 \%)$. Given that fever is a characterized symptom of SAIDs, patients seldom visited hospital for the fear of being suspicious of COVID-19, which is also featured as fever. Besides, other concrete impacts included the failure of finishing their scheduled follow-ups, the difficulty to buy medicines.

We collected self-assessment scores from patients, ranging from 0 to 10 , by which the patients were classified into three levels of disease severity: mild ( $0-3)$, moderate (4-7), and severe (8-10). The detailed proportion is shown in Table 1. The current crisis has led to a global phobia 
Table1 Characteristics of Chinese patients with SAIDs

\begin{tabular}{lc}
\hline Characteristics & Number (\%) \\
\hline Gender & $50(58.1 \%)$ \\
Male & $36(41.9 \%)$ \\
Female & $32(13-66)$ \\
Age, median, yr (min-max) & \\
Diagnosis & $34(39.5 \%)$ \\
Familial Mediterranean fever (FMF) & $11(12.8 \%)$ \\
NLRP12-associated autoinflammatory disease (NLRP12-AID) & $10(11.6 \%)$ \\
Periodic fever, aphthous stomatitis, pharyngitis, and cervical adenitis (PFAPA) & $9(10.5 \%)$ \\
NLRP3-associated autoinflammatory disease (NLRP3-AID) & $6(7.0 \%)$ \\
Tumor necrosis factor receptor-associated periodic syndrome (TRAPS) & $16(18.6 \%)$ \\
Others & \\
Symptoms & $34(39.5 \%)$ \\
Fever & $16(18.6 \%)$ \\
Fatigue or weight loss & $35(40.7 \%)$ \\
Arthritis & $24(27.9 \%)$ \\
Myalgia & $20(23.3 \%)$ \\
Cutaneous manifestations & $19(22.1 \%)$ \\
Eye inflammation & $22(25.6 \%)$ \\
Oral ulceration & \\
Self-assessment of disease severity & $55(65.5 \%)$ \\
Mild & $22(26.2 \%)$ \\
Moderate & $7(8.3 \%)$ \\
Sever & \\
\hline
\end{tabular}

$y r$ years, min minimum, $\max$ maximum called coronaphobia, raising concerns about mental health of the public [4]. With regard to psychological aspect, a total of $41(47.7 \%)$ patients stayed calm, 28 patients $(32.6 \%)$ felt worried, $4(4.7 \%)$ patients were nervous, and $3(3.5 \%)$ suffered from fear.

Although COVID-19 outbreak has been preliminarily controlled in China, the pandemic is still raging all over the world, raising grim problems. To the best of our knowledge, this is the first survey about the situation of disease management of patients with SAIDs in China since COVID-19 outbreak. Although the majority of our patients stay stable during the pandemic, some patients still had suffered from characteristic symptoms of SAIDs, such as fever and arthritis. Significantly, more than $60 \%$ of our SAIDs patients stated that their disease managements were impacted by the current crisis. Through this study, we found the low rate of follow-up was worthy of notice. Patients were more willing to complete follow-ups by visiting hospitals than through E-mails. Over half of these patients were affected psychologically, and were subjected to negative emotions. In conclusion, we suggest rheumatologists pay close attention to the unmet medical need for SAIDs patients in the unprecedented situation of COVID-19 pandemic.
Acknowledgements We appreciate all support and appreciation of the patients.

Author contributions All authors contributed to the study conception and design. Material preparation, data collection, and analysis were performed by $\mathrm{Na} \mathrm{Wu}$, Mengzhu Zhao, and Min Shen. The first draft of the manuscript was written by $\mathrm{Na} \mathrm{Wu}$ and all authors commented on previous versions of the manuscript. All authors read and approved the final manuscript.

Funding This work was supported by the Natural Science Foundation of Beijing (Grant No.7192170); the Chinese Academy of Medical Sciences Innovation Fund for Medical Sciences (CIFMS) (Grant No. 2017-I2M-3-001); the Peking Union Medical College Hospital Foundation for Distinguished Young Scholars (Grant No. JQ201705); and the National Key Research and Development Program of China (Grant No. 2016YFC0901500; 2016YFC0901501).

\section{Declarations}

Conflict of interest The authors declare that they have no competing interests.

Ethics approval This study was performed in line with the principles of the Declaration of Helsinki. Approval was granted by the Institutional Review Board of the Peking Union Medical College Hospital. (Date April 9th, 2020/No. S-K1148). 
Consent to participate Informed consent was obtained from all individual participants included in the study.

Disclosure There was not any editing agency involved in editing and submission support.

\section{References}

1. Guo YR, Cao QD, Hong ZS et al (2020) The origin, transmission and clinical therapies on coronavirus disease 2019 (COVID-19) outbreak - an update on the status. Mil Med Res 7(1):11. https:// doi.org/10.1186/s40779-020-00240-0

2. Bai X, Yang H, Qian J (2020) COVID-19 outbreak and inflammatory bowel disease management: a questionnaire survey from realistic practice. J Crohns Colitis 14(10):1494-1495. https://doi. org/10.1093/ecco-jcc/jjaa064

3. Mitratza M, Klijs B, Hak AE, Kardaun J, Kunst AE (2021) Systemic autoimmune disease as a cause of death: mortality burden and comorbidities. Rheumatol (Oxf) 60(3):1321-1330. https:// doi.org/10.1093/rheumatology/keaa537

4. Kelvin DJ, Rubino S (2020) Fear of the novel coronavirus. J Infect Dev Ctries 14(1):1-2. https://doi.org/10.3855/jidc.12496

Publisher's Note Springer Nature remains neutral with regard to jurisdictional claims in published maps and institutional affiliations. 